\title{
A Visual Rhetoric Study on the Plastic Pollution Issues in
}

\section{National Geographic}

\author{
Pengpeng Li \\ National Chengchi University \\ College of communication, China \\ Email:18698698151 [AT] 163.com
}

\begin{abstract}
This study uses the National Geographic as the research sample, and focuses on the analysis of the visual image of the environmental risk issue of "plastic pollution". Not only does it classify and sort out which image symbols used in media risk reproduction, but also discusses how the text uses, invokes and activates image information, combines "illustrations" with textual discussions, and analyzes its meaning production process and framework. The research conclusions show that public communication on plastic pollution issues in National Geographic by means of visual media representation and symbol construction, mainly using photojournalism and design creation to expose the social aspects of risks (phenomena and problems) to the public. Also, it presents and tells readers the reality (source and essence) of risks in a scientific and simple manner, and inform the public of the ideal aspects of risks (practical methods), and guide the public to engage and participate in environmentally friendly actions.
\end{abstract}

Keywords - environmental risk, plastic pollution, magazine, visual rhetoric, perception of risks

\section{FORMATTING OF MANUSCRIPT COMPONENTS}

The United Nations 2016 report pointed out that marine plastic waste has become a key factor threatening the ocean and life species (Dias, 2016), it has become the second largest environmental risk threat to mankind after climate change. In the past ten years, deaths of marine species due to accidental eating of garbage or being entangled by garbage have increased by $40 \%$. Among them, plastic products are the deadliest marine killer (Dias \& Lovejoy, 2012). The public's perception of risk is different from that of scientists and professional elites, and the formation and impact of environmental risks are hidden and diverse. Mass media serves as an important link to construct public risk perceptions. How to communicate risks with the public, and how its strategies and effects are always the focus of attention of environmental communication researchers.

Natural and environmental themes are visual in nature, and human perception and understanding of environmental risks are more likely to be affected by the visual space created by the media (Wahlberg \& Sjoberg, 2000), to a certain extent, visual images help or hinder people from taking action (Meisner \& Takahashi, 2013). David Perlmutter (1998) pointed out that images can stimulate the audience's emotional response, and clear content presentation can promote the emotional connection between the communicator and the audience. Aarti Iyer and Julian Oldmeadow (2006)also confirmed this theory, that is, comparing textual information with visual images, individuals will have greater mood swings when viewing visual images. Because the communicator's control over the rhythm of the composition, color, and elements of the visual picture, it is easy for the audience to produce emotional follow and movement effects, which in turn produce emotional perception effects. In other words, the use of image symbols in visual communication is of great significance to the shaping and communication of environmental risks. However, in academic research in the field of environmental risk, there have been relatively fruitful research results on the language rhetorical framework of media reproduction of environmental risks, but there are few analyses of visual communication and rhetorical frameworks (Hansen \& Machin, 2013; Simonsen, Bærenholdt, Büscher, \& Scheuer, 2010). For example, scholars such as Liisa Antilla (2005) and Carvalho (2005)When analyzing the scientific reporting framework and themes of " climate change " in American newspapers, it is concluded that in the analysis of media representation in the academic world, images are often used as supplementary research in text analysis, and have not become the focus of research and discussion. But for the construction of risk issues, images have an influential force that cannot be ignored. 
As a media carrier that emphasizes visual beauty and visual persuasion, magazines are published faster than books, and their content editing is wider and deeper than newspapers. At the same time, they combine the advantages of newspapers and books, and avoid the publishing speed of the two, and layout restrictions, has an indispensable influence in the field of communication. However, compared to newspapers, television and Internet media, " magazines " pay relatively little attention to environmental communication research(Adam, 1998). Newspaper media has always been the main carrier of environmental risk content analysis, and there are more prominent research results in both textual narrative and visual rhetoric (see, e.g., Anne DiFrancesco \& Young, 2011; Howenstine, 2005; Smith \& Joffe, 2009). Check the domestic and foreign research literature on the reappearance of environmental risks in " magazines ", and only find academic articles that use "National Geographic" to study its text and visual images (see, e.g., Ahern, Bortree, \& Smith, 2013; Remillard, 2011; Todd, 2010), and the number is small. It can be seen that the rhetoric and image research of environmental geography " magazines " are more likely to be ignored by environmental communication scholars. Even if there are some research outputs, they mostly focus on the social phenomena constructed by visual images, and fail to analyze the " magazines " . "The image composition, symbolic metaphor, the use of visual rhetoric framework and how to effectively convey the risk message to achieve the persuasive effect. However, environmental geography " magazines " not only have the objectivity and scientific nature of newspaper content editing, but also have high image quality, layout design, and reading rhythm arrangement, which can more intuitively disseminate natural and geographic environmental affairs to the public. The analysis of visual rhetoric and image symbols in environmental geography " magazines " can help environmental communicators, environmental protection workers / ecological artists have a deeper understanding of the characteristics, advantages and necessity of environmental risk visualization, and then in the future communication design work Organize more effective visual symbol information and visual strategies to communicate with the public.

In summary, this study uses the English version of the scientific geography magazine "National Geographic" as a research sample, and focuses on the visual presentation of the environmental risk of " plastic pollution ", focusing on how the selected image symbols construct risk information and how to persuade The public attaches great importance to plastic pollution and participates in actions, while observing how the traditional Chinese version is translated into the English version. The research purpose and expected contribution mainly have three points:(1) Explore the characteristics and trends of the construction of visual symbols for plastic pollution in Scientific Geography; (2) Using semiotics and visual rhetoric to describe the visual symbolic language and rhetorical framework constructed by the magazine, at the same time, to propose a path for the academic integration and dialogue of visual communication design and environmental risk communication; (3) Analyze the methods to effectively enhance public awareness and environmentally friendly behaviors, and hope that this research will provide enlightenment for visual design, image analysis and public communication of environmental risks.

\section{LITERATURE REVIEW}

\subsection{Characteristics and reproduction of environmental risks}

In the 1980s, the German sociologist Ulrich Beck proposed that the industrial revolution and technological rationalism not only gave birth to the state of a series of environmental problems and social structural imbalance, bring a lot more unpredictable and difficult to human society and the environment The potential risks of control, Hyundai is in the structure of a risk society (Beck, 1992; Yang \& Hsu, 2012). Beck (2002)believes that risk is a cognitive system at the same time, with a high degree of " uncertainty " and artificially constructed meaning, representing a potential threat and disaster. The complexity and uncertainty of the risk society make the public's perception of risk different, and the public's interpretation of risk can often affect their participation(Yang \& Hsu, 2012). If you do not reflect on the discourse and practical behaviors that lead to various environmental risk threats, And to rebuild the relationship between man and nature, both man and nature will be deeply trapped in repeated cycles of risk damage(Yang \& Hsu, 2012).

As far as the concept of risk is concerned, it refers to the possibility of occurrence in the future, so risk is an idea, not a fixed reality (Kinsella, 2002). People may know the probability of risk formation, but they cannot know when the risk will occur and what behavior will accelerate the formation of risk or even crisis. On the one hand, environmental risks are concealed and diverse. Many environmental issues are not easily described directly and are relatively invisible(Johnson \& Covello, 2012).This brings many difficulties and obstacles to media workers' agenda setting, text reproduction (including images and text), and public communication. For example, when the media reproduces scientific and professional risk topics such as " climate change ", " nuclear radiation ", and " marine pollution ", it is difficult to truthfully understand the formation and impact of risks due to the limitation of the media's own professional knowledge and balanced reporting positions. Present to the public. Moreover, many environmental problems are not clearly manifested, and the substances that cause environmental problems may not be seen by the public or appear to be harmless (Peeples, 2011). For example, a large number of plastic products in marine pollution will form plastic particles when exposed to the sun. Such particles can stay in water for thousands of years and are not easily degraded. Scientists even claim that washing cosmetics contain a large number of plastic particles, which are easy to cause. Water pollution threatens the survival of species and the 
ecological environment(Kershaw, 2016). This kind of highly concealed professional risk knowledge, through simple visual elements to show the reasons and consequences covered by the risk, and to be understood and recognized by the public is a challenging task (Doyle, 2007; Dunlap, Bechtel, \& Churchman, 2002; Pezzullo \& Cox, 2017). On the other hand, environmental risks have timeliness and uncertainty. Schoenfeld's (1979) study, it was pointed out that the long-term and slow evolution speed of environmental issues contradicted the rapid operation mode of the news reporting cycle, and thus became the main challenge for media reporting. Environmental risks also involve the operation of natural ecosystems. Among them, the operation of the ecosystem itself has a high degree of uncertainty. There is no research that can really grasp the internal operation laws of nature, and all methods for estimating and predicting the development and changes of natural ecology are also uncertainty. This discussion of uncertainty further complicates risk information that is already difficult to understand and hinders public perception and behavior. These characteristics make media reports and visual representations of environmental risks vulnerable to manipulation by man-made ideologies and misunderstandings at the personal cognitive level. Of course, human beings have long realized that the perception and image interpretation of the environment are affected by many aspects such as social background, political system, and cultural construction (Macnaghten \& Urry, 1997; Soper, 1995; Urry, 1992; Williams, 1975). Specific discourse structures and natural representations are selected by different ideologies and used in various media communications, such as product advertisements, ideas and marketing strategies, tourist city publicity, TV documentaries or movie presentations. With the continuous expansion and deepening of research fields, research cases on the use of visual elements and rhetoric have gradually appeared. Cottle (2000)study of television news coverage of environmental analyzes, he learn from Roland Barthes semiotic theory, emphasizing the ecological perspective vision of romanticism, the natural environmental performance as a landscape, this The landscape is undergoing destruction and unknown threats. However, the details and process of such environmental destruction and threats are not presented. Therefore, constructing environmental risks and promoting public communication through visual images and rhetoric is not just a static depiction, but a process of continuous development and change (causes, influence, prevention / blocking).

\subsection{Symbolic narration and rhetoric of environmental communication}

Stanley Baran (2011)believes that symbols mediate or control the public's perception and imagination of the external world. The public, as image consumers, constantly associate socially-designated image symbols with specific environmental issues or phenomena over time. For example, when it comes to climate change, people often associate images with melting glaciers, polar bears and penguins, extreme natural disasters, etc. ; When it comes to air pollution, one thinks of smog, automobile exhaust, and smokestacks in industrial processing plants; nuclear pollution thinks of mutant plants and deformed babies; when people think of marine pollution, people will think of oil spills, Marine / coastal garbage, these images may not be able to show the full picture of the risks, but they can provide the public with visual clues and image memories, and at a specific point in time become a public vocabulary with recognizable, communicable meaning and universally recognized by the public. Pierce that, the operation symbol having a symbol system such that the image anchor and the relay unction, the public towards meaning preset image producer and by means of which a series of complementary The image fragments of the relationship convey the complete content of the message (Barthes, 1977).In order to achieve an impact on public behavior. From the behavior involved in perspective, George Mead think that " self " is divided into human cognitive level " lord " and " off my " two parts, the main I was impulsive, full of desire, unconstrained, And the guest self is restrictive and restrictive, and the expectations are consistent with the expectations of others. When making certain behaviors and decisions, people often consider both their own impulses and the evaluation of others after the behavior occurs (Mead, 1934). However, the symbolic narrative of environmental risk has limitations in its impact on the public " object-self" presentation.

According to the Smith and Joffe's (2009)research, image symbols provide the public with only a one-sided and narrow focus. While providing visual clues to the public, they also frame the public's cognition and imagination, although realistic and disastrous images will risk factors for a clear threat to the public daily life, it is difficult to see how to deal with the public and address the risk; and The study of Anne and Young (2011) found that images and language texts exhibit different dimensions through careful analysis of the interaction between images and language texts, that is, images do not actually drive the interpretation of the content of the article, and language texts do not really convey the meaning of images to The public, in other words, the images and language texts have not played a role of mutual reinforcement and complementation. On the other hand, articles with pictures and texts contain more moral and emotional dimensions than single-language reports without images. The connection between image symbols and personal emotional senses can trigger public participation in environmentally friendly behavior and decision-making (Joffe, 2008). However, the overall graphic narrative structure does not provide technical arguments for causality. This means that when analyzing the meaning production process of symbols, we need to pay attention not only to the basic composition and combination of image symbols, referential relations and meaning mechanisms, but also to the use of image symbols in specific phenomena and their possible impact effect (Liu, 2018). Exploring whether the image narrative and meaning production system within the visual rhetoric framework have limitations, and whether the interaction between language and image symbols can effectively and effectively play the role of persuasion and recognition in risk communication.

Liu Tao (Liu, 2016)emphasized that visual rhetoric always follows certain framework rules. The framework provides 
a semantic production system to guide and restrict the public to approach, perceive and understand phenomena or things in the way preset by the producer (Liu, 2014). And according to the different social backgrounds and cultural concepts, the information is coded, combined and arranged, and finally presented is a more perceptual, intuitive and concise visual cognitive system, in order to achieve the purpose of a visual closer to reality (Liu, 2018). Different from semiotic research approaches, the biggest feature of " visual rhetoric " is the attention to the effect of the rhetorical frame. First, the visual rhetoric frame gives the content drama and expressive tension in the process of meaning production; secondly, it is persuasive Activate and combine sexual rhetorical image or cognitive image (Hill \& Helmers, 2012). On the other hand, any sign system has a rhetorical cognitive dimension, and its meaning is to explore the meaning production, operation rules and deep " rhetorical structure " of image signs. This rhetorical structure implies a kind of symbol encoding. The process of narration and persuasion is realized through the rhetorical framework of metaphor, metonymy, irony, and symbol (Liu, 2017).

Sonja Foss that, provided the visual analysis of the frame element is the need to classify the visual encoding, via for " color space, the structure of " regularity of the visual elements and the like, constituting the inductive rule and summed image inside the symbol (Berger, 2017). Foss once proposed a problem-oriented visual rhetoric model based on rhetorical effects. It mainly includes three steps: First, place the image symbol in a specific communication structure, based on the physical attributes and characteristics of the image ontology, and identify the " function of the image symbol " " , that is, the original intention of the image symbol's function; secondly, comprehensive observations are made from the subject, medium, material, form, color, composition, and external environment of the image symbol, and the meaning of each dimension to the image " function " is evaluated. What kind of influence does the method have; finally, starting from the original intention of the image, distinguish the legitimacy and rationality of the image's " function ", such as whether there is ethical and moral justice in the construction of the image symbol system (Berger, 2017; Liu, 2017). The Foss This method of analysis, although emphasizes rhetorical effect, but it ignores the significance of visual symbols of social history behind the production system and ideology, there is no established link between the rhetoric from the visual to the visual grammar of discourse (Berger, 2017). Rice (2004) believes that all inductive and deductive reasoning methods are based on adductive reasoning, emphasizing the " visual observation " of things, which exists as a kind of origin of perception and cognition, and can connect the brain with the previous possible existence of things At the same time, when expounding visual rhetoric works, Rice put forward four main analysis indicators, namely oppositional elements , including conflict, dislocation, juxtaposition, irony, contrast, etc.; isomorphic elements (co-constructed elements), emphasizing the interaction between the text and the public, such as ambiguity, realism, surrealism, simulation, participation, integration, etc.; contextual elements, explaining how the text and the language environment pass through various possible symbols. Produce relevance effects; ideological elements, emphasizing the details of how visual rhetoric works are politicized, personified, durational, and anti-objectification, aiming to show that visual rhetoric analysis not only needs to identify four elements in the text content, And analyze the content, form, and visual experience fusion corresponding to these four elements. Liu Tao integrated the above-mentioned scholars' analytical methods of visual rhetoric works. Liu Tao emphasized that visual rhetoric framework must have two basic premises, namely rhetorical purpose and problem context, in order to ensure the scientifically of visual rhetorical framework research. Among them, rhetorical purpose dominates the direction of visual framework. And the concept; the problem context determines the perception object and interpretation space of the visual frame(Liu, 2016).

Then, when using the theory of signs and visual rhetoric to analyze the visual representation of environmental risks in magazine texts, are there any analysis methods and principles that have not been discussed by scholars? In the combination and construction of visual elements, how does the image realize the meaning of words and is the interpretation of the image appropriate? In what form does the rhetorical frame affect the audience's perception and behavior?

\subsection{The visual rhetorical framework of environmental risks in the magazine}

Compared with newspapers, magazines have lower distribution frequency and lower timeliness, so they are generally ignored in communication studies (McQuail, 1987). However, magazines have higher printing quality, rich graphic content, clear categories, high record-ability and preservation, more flexible and free layout than newspapers, and higher reading autonomy (including time and location)., Method), through the unique form of expression and excellent visual perception, the persuasive effect can be achieved more effectively (Li \& Wang, 2015; McQuail, 1987). After the environmental movement in the 1960s , magazines began to pay attention to environmental issues, and academic research on the reproduction of environmental issues in journals appeared. Messner and Bruno Takahashi (2013) use the quantitative and qualitative comprehensive analysis for "Time" magazine, presented the main elements that make up the four environmental issues: Environmental symptoms (such as climate change), causes (such as excessive burning of fossil Fuel), participants in environmental debates (such as environmentalists), and solutions (such as setting stricter fuel usage requirements). However, when analyzing the framework of its visual rhetoric, they found that the cover of "Time" magazine tends to be photographed way concerned about the unintended consequences of human activities caused by the (climate change, species extinction, pollution), without showing actual human activities, such as to obtain, consumption, waste and other damage to the environment, suggesting that "Time" magazine has neglected presentation of the root causes of environmental problems. Although there are detailed analysis of the causes and results of environmental problems in the 
article, they are not fully displayed on the cover image. In the metaphorical analysis part of symbols, the signs of environmental pollution in the "Time" magazine mainly include Animals (oil-soaked birds, skeleton fish), pollution sources (mushroom clouds, nuclear reactors), humans with painful expressions, and deterioration of the ecological environment (dense smoke, oil floating on the sea, melting ice), but the text is not included on the cover It is anchored in the interpretation of the meaning of the image, and some covers only use text as the main image symbol. With reference to this research, we can see that visual symbols or words alone cannot fully express the integrity of environmental issues, because the connotation and extension of the symbols themselves can be very extensive, and they need to be elaborated with words to allow the public to correctly interpret the content production. The real intention of the reader, and accurate image presentation can enhance the perception, experience and memory of the content. Therefore, when conducting risk communication on environmental issues, it is necessary to pay attention to the rhetorical framework and effects of text and image symbols at the same time.

\section{RESEARCH SAMPLES AND ANALYSIS}

\subsection{Sample}

This study uses the " National Geographic " as a sample, and analyzes the visual symbols and rhetoric of the special report text (including images and text) on the theme of " Plastic Pollution " published in June 2018. The special report occupies a total of 50 pages. , A total of 31 image texts. The cover of this issue uses " PLANET OR PLASTIC " (Figure 3.1.1) as a metaphor " the plastic hazards you see are just the tip of the iceberg ". The special issue uses photographic pictures, charts and graphic design works in various forms to present the current situation to the public. The threat of plastics facing society and species, an in-depth analysis of the damage caused by plastic waste to the marine environment and species, aims to awaken the public's attention to plastic pollution and environmentally friendly behavior. By analyzing, summarizing, and categorizing each sample, this research observes how the " magazine ", a communication medium that is different from traditional media, uses symbols, graphic narrative structures, and visual rhetorical frameworks to spread risk issues, thereby giving full play to the public Persuade function.

Figure 3.1.1. PLANET OR PLASTIC

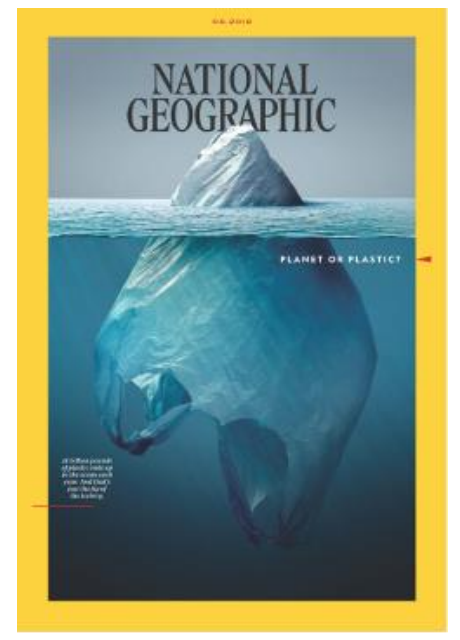

Figure source: National Geographic, June, 2018

\subsection{Textual Anaysis}

\subsection{1 "Perception " framework for plastic pollution}

The cover of the magazine is typeset vertically, and the main vision is framed with a yellow frame. The picture composition is mainly pictures. Others such as the name of the magazine, the theme copy, and the publication date are integrated into the main vision for typesetting, and placed in the frame with the main vision (As shown in Figure 3.1.1).

First of all, bright yellow is the iconic color of "National Geographic", and it is also the uniform color of all editions of its global release. It symbolizes that the magazine's goal is to open the "window " to the world for the public . At the same time, this color is different in skin tones, Readers of different cultural backgrounds and different languages gradually build up memories of exploration, classics, quality and passion. From the perspective of consumers' cognitive psychology, compared with products with complex, changeable, and non-brand consistent logos, simplicity, purity and visual impact are a cognitive habit of consumers to establish identity with products and brands. Secondly, the magazine title " 
NATIONAL GEOGRAPHIC " adopts the lowest lightness black font, which forms a perspective contrast with the high lightness foreground image " Iceberg ", and the ocean and " Iceberg " are located in the center of the visual image and account for the largest proportion. By means of visual metaphorical rhetoric, The public complained that plastic shopping bags, a common plastic waste that spreads in people's daily lives, are just the tip of the iceberg in the current ocean full of plastic trash. It aims to highlight the theme of this issue - plastic pollution, which is also "National Geographic". For the first time, the focus is on a risk spread of " plastic pollution ". Third, while the image is focused by the frame, its meaning is also "anchored" by the text (the title and description of the main text). The first-level guiding symbol "Red Triangle Arrow" on the high-light yellow frame leads readers' attention to the title of the main paper "Planet OR Plastic". And then, the description of "18 billion pounds of PLASTIC ends up in the ocean each year. And that's just the tip of the iceberg" by the second-level guiding symbol "Red Line", to stimulates readers' perceptual experience. Ask the reader to think about what happens when so much plastic waste gets into the ocean system. In both visual and meaning production, National Geographic uses image focus and preset symbolic meaning to enhance the graphic coding features of information transmission and public communication (communication purpose).

The construction of the perception framework in the article includes two types of photographic images (realistic pictures) and design drawings (artificial combination and processing), which are deconstructed and reorganized with realistic photographic techniques and abstract visual elements to focus, record and spread plastic pollution. And hazards, and give visual works a frame of meaning through text description. Regardless of the coding of the cover graphic or the graphic characteristics of the inner page, the role played by the text can guide the reader's possible interpretation of the image symbol to the specific dissemination content (plastic pollution) and purpose (risk communication) (Barthes, 1977). The picture composition, combination and frame analysis are as follows:

\subsubsection{The photographic presentation strategy of "plastic pollution"}

Photography (realistic photos) is very important for the focus and positioning of readers' topics. High-angle / longdistance photography and composition methods and the content / messages to be described are large-scale and panoramic, creating a potential empowerment sense (Peeples, 2011), such as aerial photography. Of course, this kind of photographic technique provides the public with a new and unusual perspective, a brand-new way of looking at the object, which is usually not accessible or seen by the public normally / usually (Elliot, 2013). In contrast, low-angle / close-range viewing angles and macro close-up shots emphasize a sense of closeness and careful observation, which can better grasp and understand the essence of the phenomenon, and are more suitable for the high concealment and uncertainty of environmental risks. Disseminate sexual issues and communicate with the public. The photographic images in the textual adopt close-up observation photography techniques to guide the reader's attention to the content narrative structure preset by the image producer.

Photographic narrative can be divided into two kinds: individual image content statement and series image comprehensive narrative. Individual images emphasize the objective record of "pollution degree", but they are not only the reproduction of "landscape" (phenomenon). "people"/" animals "elements will be integrated into the photos, and the visual center will be set through the cutting of the picture, the contrast of elements, and the rhetorical methods of conflict/contradiction. First of all, photographic images with human elements are integrated. The description of plastic waste recycling industry in Figure 3.2.1.1-3.2.1.4 on the following page shows that "plastic recycling" has become an informal "business" for the reader, showing the current situation of economically backward areas and the huge volume of plastic waste. Figure 3.2.1.5 uses a series of images and a little composition method to show the public the retail enterprises of plastic products, aiming to tell the readers that plastic products have been flooded in our daily life. Figure 3.2.1.6 shows the "Disposable Life" from Life magazine in 1955, which tells us that disposable plastic products have become a necessity for daily Life, but today are stifling human society and species. Second, into the animal elements of photographic images, as shown in figure 3.2.1.7 a close-up of the hippocampus, caption "hippocampus for drifted in the current forward will take pieces of seaweed and other natural objects, however, is now caught a cotton bar", this kind of unusual "intimate relationship" with the public daily necessities "cotton buds" buckle even mutually, intentions to make people think about their behavior could pose a threat to Marine species. Figure 3.2.1.8 highlights the global nature of the risk by using a collection of photographs of plastic pollution from cities in different countries (Ethiopia, Japan, Spain) to recreate the damage caused by plastic waste to species in different countries and regions. , it is worth noting that photography presents four forms: content and descriptive text text on the image (figure 3.2.1.7), but as a square image and images form the composition is balanced, attracted by visual images in the readers of the moment, guides the reader to find descriptive text, and therefore this form emphasizes the readers' reading actively; The text is located around the picture (Figure 3.2.1.4, 3.2.1.6), and the text is only used as a supplementary statement, which is weakened in the overall picture. The reading of the text content mainly depends on the guidance of the image. This arrangement rhythm is to introduce the reader's vision from the image to the text, to answer the readers' questions about the image content. Text as part of The image (figure 3.2.1.5), The color images in low lightness of pure color, highlight The main picture, The title font for enlarging words with tall lightness images motif "The World Capital of Everyday Plastic", supplementary text to illustrate The universality and particularity of Plastic products (large), will people ignore daily, seems to be insignificant "Plastic" with photography technique brings readers suffocation visual sensory experience, and to construct The "Plastic damage" cognitive aspects 
elaborate; A text description of the image and the layout of all images of descriptive text collection together (figure 3.2.1.8), or the text and the integration of image interpretation on the next page, its purpose is to expect the expression of visual sex, let the text, on the one hand, emphasize the reader's initiative, looking for relevant text explanation, on the one hand, in words to the picture to watch again, and after reading the text explanation will be deeper and longer on the image memory and perception. No matter which form, it is intended to emphasize the mutual drive, guidance and activation of pictures and texts, so as to bring readers into the context of content narration, humanistic care, social observation and exploration (Gamson \& Modigliani, 1989). It highlights that human beings live in a "self-inflicted" risk society (Chou, 1998). If we do not timely reflect on the behaviors that lead to such environmental risks and rebuild the relationship between human beings and nature, both human beings and the natural world will be deeply trapped in the repeated cycle of risk damage (Kinsella, 2002).

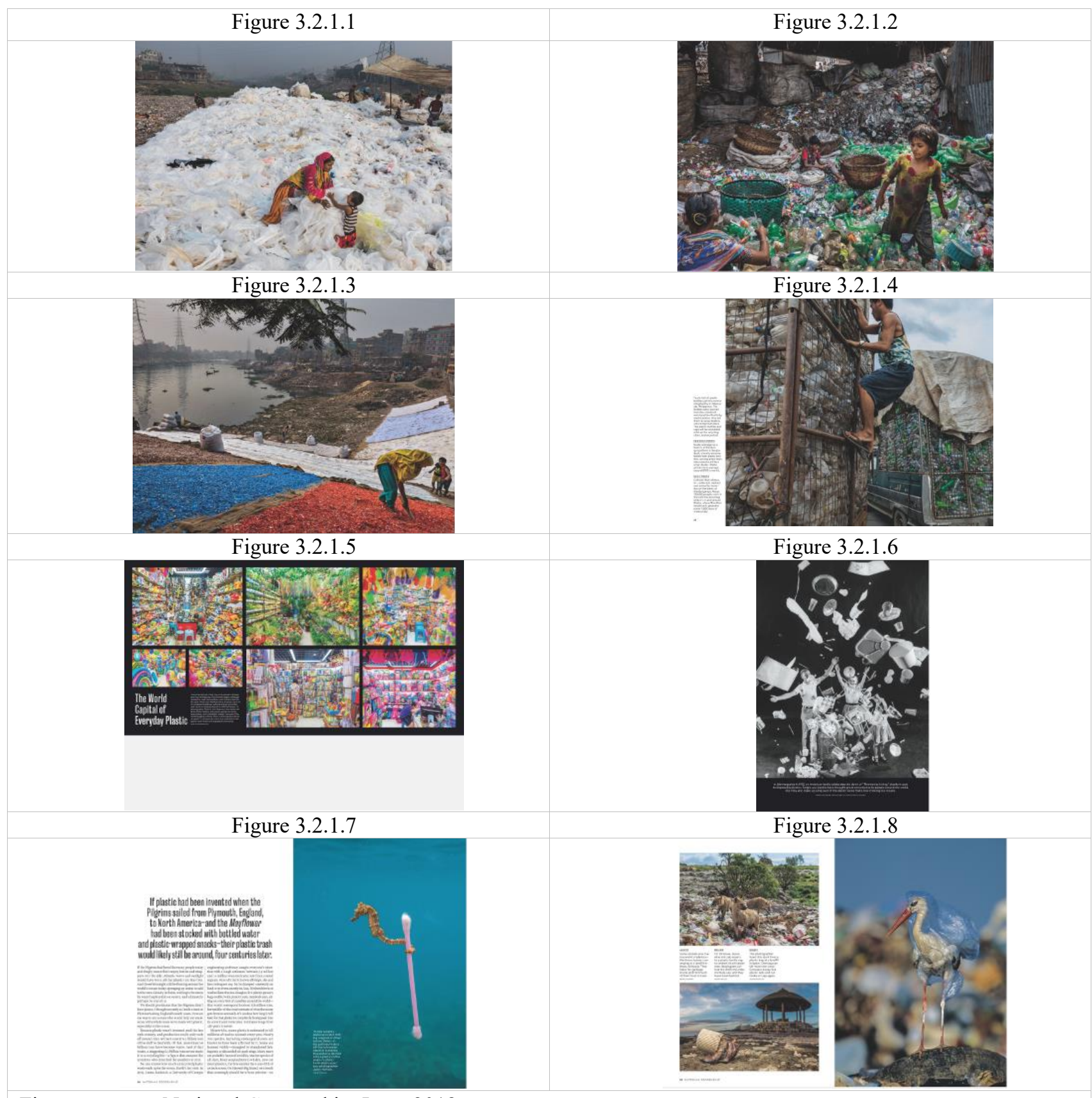

Figure source: National Geographic, June, 2018

\subsubsection{The Visual design presentation strategy of "plastic pollution"}

Magazine within the text layout designer for " plastic pollution " Art and Design processing screen performance issues, and " Design "and " Art " is the biggest difference is that " Art " is more self-expression, And " Design " emphasizes being understood by the audience. In other words, designers need to understand how information may be interpreted and what kind of visual cognition or emotional experience may be brought to the audience. Figures 3.2.1.2.1 and 3.2.1.2.2 take 
"Plastic·Art" as the title. Through the reconstruction and deconstructive design expression of the " plastic " element, it is a metaphor for " plastic is everywhere " . Figure 3.2.1.2.1 uses a low-brightness large-area background color to highlight the main vision or design theme, adopts a " one-point " composition, and places the core image elements at the best visual point of the screen, and then uses a combination of perspective elements to guide the audience Visual attention extends outwards, and then reads linear text titles and copywriting, and then repeats reading images to complete a complete visual narrative.

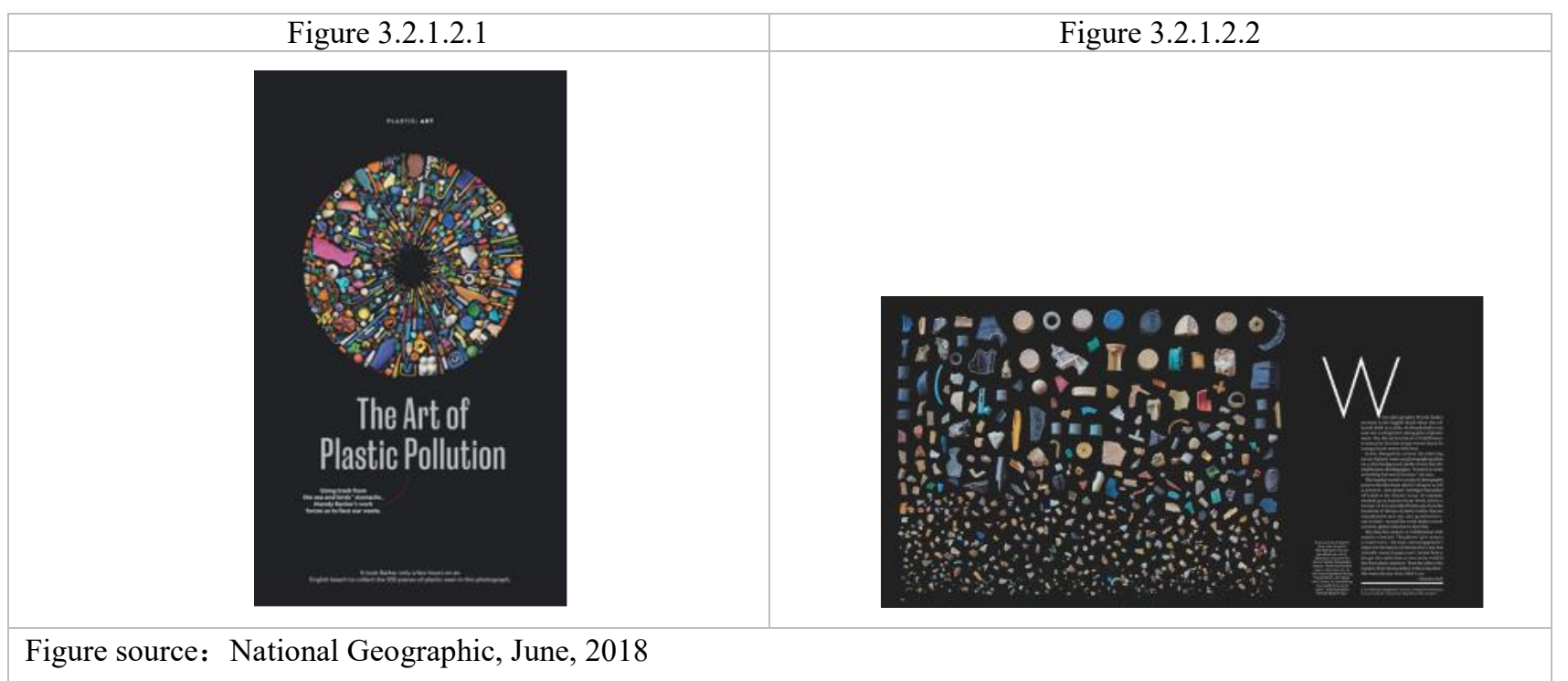

It is important to note that the figure 3.2.1.2.1 at the bottom of the document in the form of "line", told the audience on the image elements are from the bird's stomach, cause the audience in the perception level of curiosity, and then read the next section, and figure 3.2.1.2.2 uses a flat way of composition, use 1.5 pages to occupy all the audience visual space, to achieve "shocked" sensory experience, text content through the "capitalize the first letter" and "zoom in" mode of processing, guide the audience in strong shock after the experience of reading text explanation, to better achieve visual persuasion; figure 3.2.1.2.3 on the next page simulates the state of ocean whirlpool with the arrangement of visual elements, and the elements are all from real materials. While shocking the audience, the audience's "gaze" on the picture aims to achieve cognitive persuasion by creating a "reflection vortex". Figure 3.2.1.2.4 shows the global journey of Coca Cola with plastic bottle caps in the form of a sketch. The main vision is located in the center of the picture, and then the lines are used to explain. This ordinary visual narrative technique tells the audience that "plastic is everywhere" and the global risk of "plastic pollution".

\begin{tabular}{|c|c|}
\hline Figure 3.2.1.2.3 & Figure 3.2.1.2.4 \\
\hline 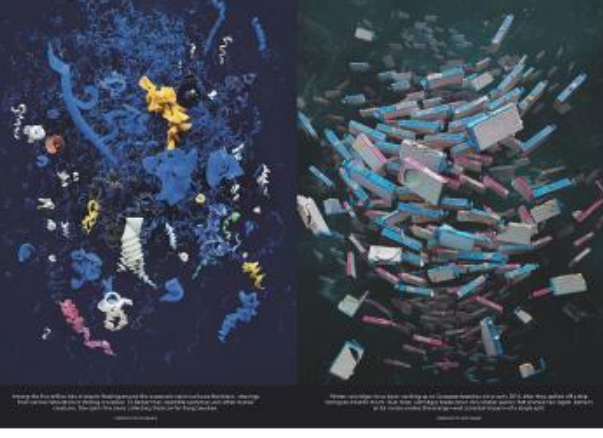 & 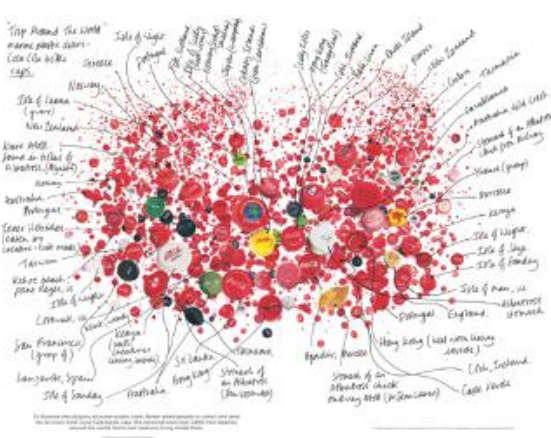 \\
\hline
\end{tabular}

Figure source: National Geographic, June, 2018

In general, the rhetorical strategy of this part of visual content is to complete the visual narrative through the arrangement of visual clues, the composition of the picture and the expression of color. By creating the perceptual atmosphere of "shock", the audience can experience the proximity of the risk of plastic pollution, and then achieve the public communication and persuasion of the risk.. 


\subsection{2 " Understanding " framework for Plastic Pollution}

As mentioned in the literature discussion, visual symbols or words alone cannot fully express the completeness of environmental issues, because the connotation and extension of the symbols themselves can be very extensive, and they need to be explained in words to allow the public to correctly interpret the content production. The real intention of the reader, and accurate image presentation can enhance the perception, experience and memory of the content. Therefore, the content of the magazine's special issue relies on the context of the source, composition, and pollution of "plastic" to disassemble risk issues and disseminate scientific knowledge. The scientific content has a certain degree of professionalism and ordinary people's cognitive difficulty. In the layout of the text, the pictures play a role in alleviating the cognitive pressure. On the other hand, as shown in Figure 3.2.2.1 and 3.2.2.2, through the visual analysis and presentation of scientific data, the audience can understand the spread of data and risks while seeing the data. To a certain extent, the uncertainty and threat of "plastic pollution" risks are brought into public perception in a visual way, and the public's lack of environmental literacy is emphasized on the impact of pollution problems, with the intention of persuading the collective interests of society public.

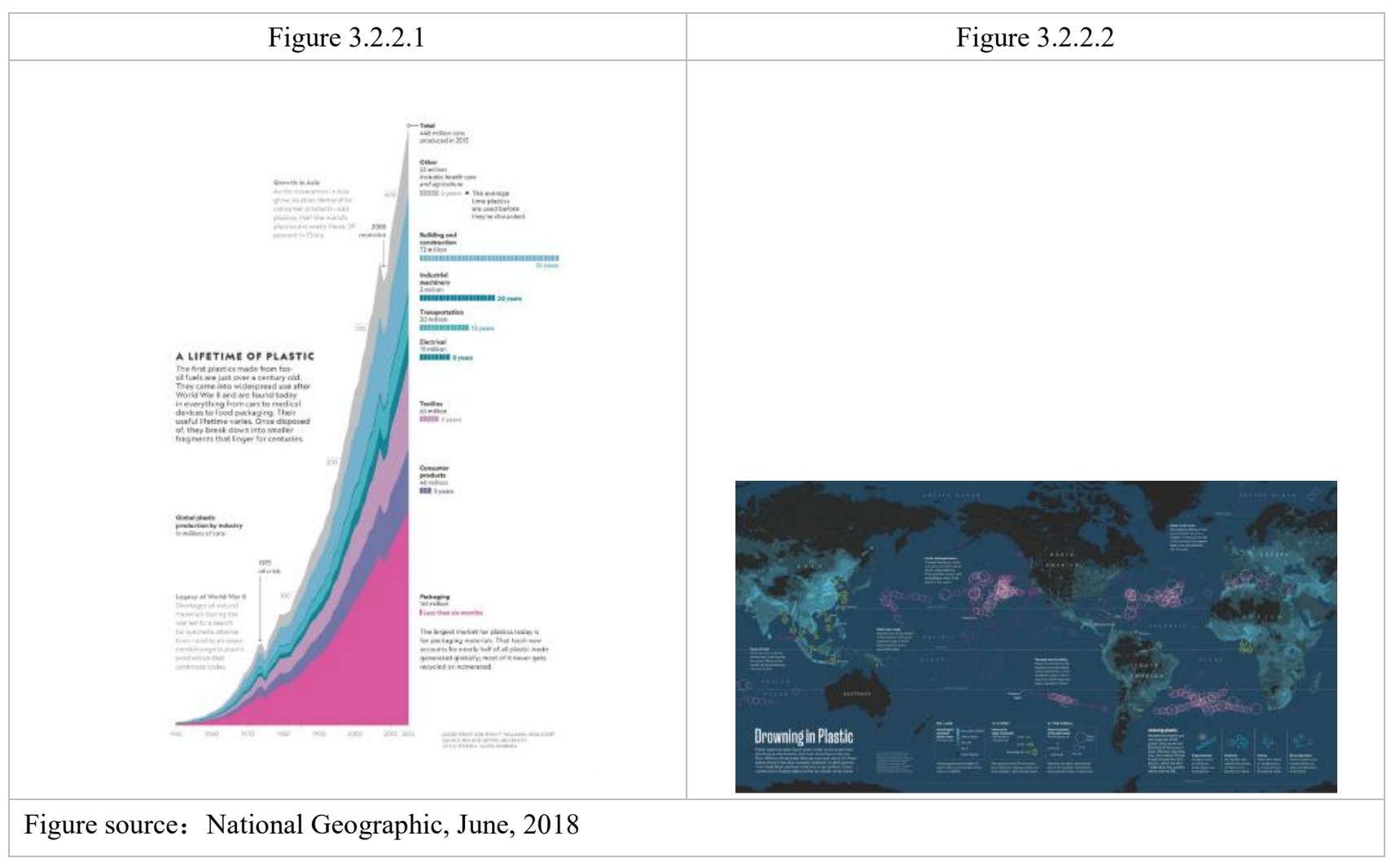

\subsection{3 "Action" framework for plastic pollution}

In the past, the dissemination of environmental risk issues mainly focused on the analysis and description of the "causes" and "impacts" of risks, and lacked the content of "how to cope with and solve". Phase and the content of the special issue on the audience cognition to the plastic pollution are everywhere, and its sources and harm, at the end of the special issue, respectively, through the analysis of the "plastic pollution" the difficulty of risk management (figure 3.2.3.1), and introduce other countries to "plastic pollution" the successful experience of risk management (figure 3.2.3.2), and to provide "us" how to start from their own coping, ease and solve the "plastic pollution" the specific measures of risk (figure 3.2.3.3) this three parts, let the audience understand the risks and solutions. 


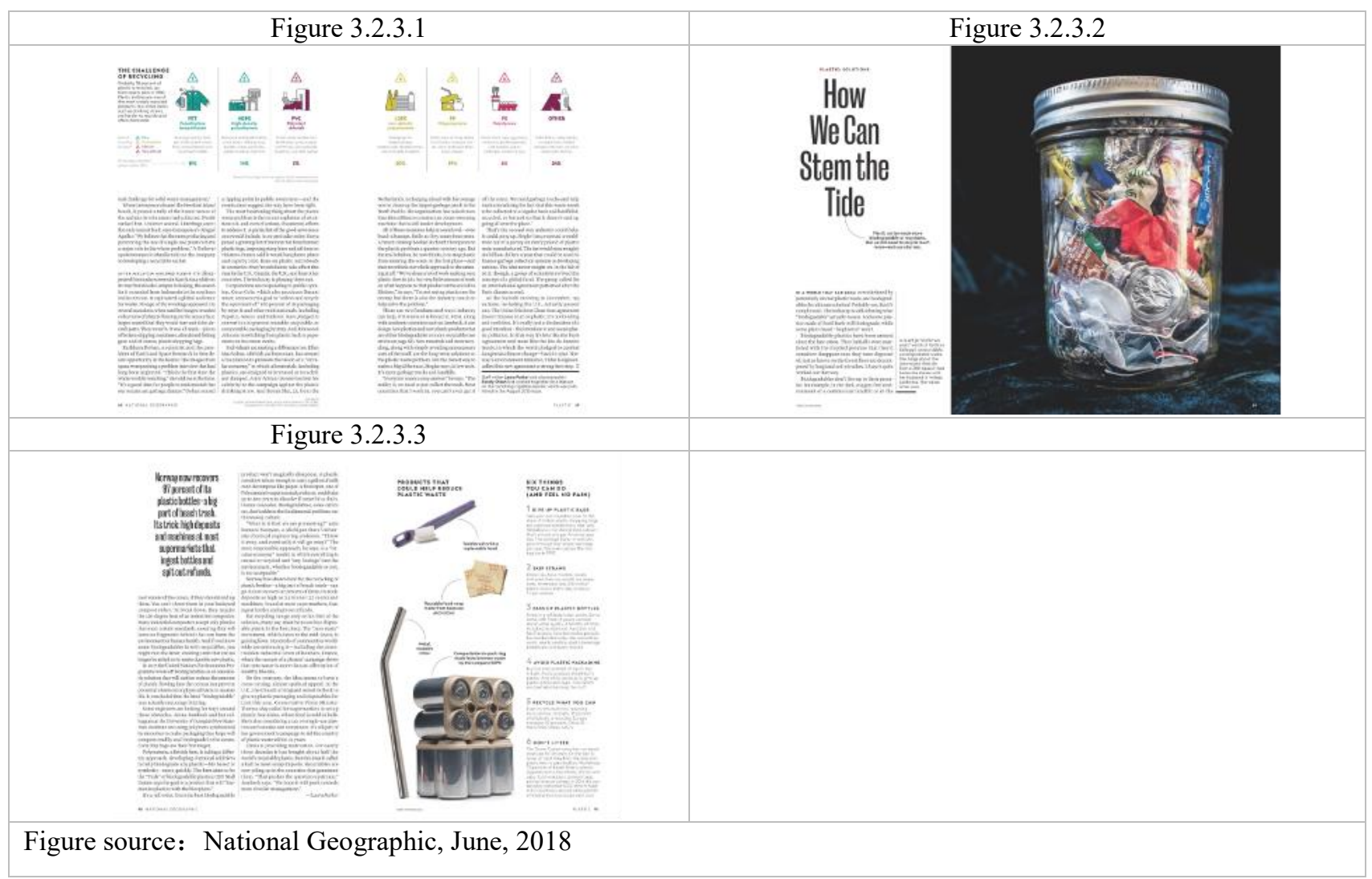

The combination of graphics and text in this part of the content adopts the traditional layout design strategy, the text surrounds the image, the design and color arrangement of the image or symbol, and the selection of flexible, jumping, and diversified language methods to relieve reading and visual fatigue Role, to drive the audience to complete the reading and reflection of the text content.

\section{RESULTS}

Based on the observation and analysis of the issue of " plastic pollution " in " National Geographic " magazine, this research believes that the magazine's symbolic narrative and visual rhetorical framework for environmental risk can be summarized as perception (epistemology), understanding (ontology), and action ( Methodology) Three parts.

First, at the perception level, the "epistemology" that shapes the knowledge system refers to the shaping of the public's perception of the risk of "plastic pollution", so that the public can see the appearance of the risk and the harm it has caused, and at the same time it can be seen The responsibilities and normative framework of media reporting are not to passively present what the public "want to see", but to actively "let the public see" the current social situation, which in turn arouses the public's attention to the issue. In the construction of the visual meaning of the magazine's plastic pollution issues, the pictures play a leading role in guiding and explaining. Through a large number of photographic pictures and visual design works, the public can see the problem of excessive plastic products and plastic waste. And through the concentrated, fusion, and flat visual composition processing; the contrasting presentation of the foreground and background in color brightness and purity, and the visual clue arrangement of the graphic design in the point, line and surface, the intention is to bring the audience "shock". The aesthetic effect and topic thinking of the audience immerse the audience in the perceptual atmosphere of "plastic everywhere", with the intention of achieving the purpose of "silent rhetoric". Create a normative framework at the level of social collective interests, using visual rhetoric strategies of "depoliticization", "artistic" and "shock aesthetics" to stimulate public thinking about the social environment, their own behavior and actions. This kind of framework is often more stimulating to the public's awareness of participation in behavior than those that use fear rhetorical strategies (such as extinction, deformed babies, smoked chimneys, etc.) visual issues, although fear images may attract visual attention The level is effective, but such images cannot stimulate the public's rational thinking, and tend to make the public's risk assessment of the issue in a weak and numb state, which is not conducive to the public dissemination of the issue..

Secondly, at the level of understanding, describe the " ontology " of knowledge . Risk issues are truly presented in the public's field of vision. The next step is to let the public have a deep understanding of the causes, structure and hazards of " risks", " knowing what they are, and knowing why", so as to improve citizens' ecological literacy, and trigger the public's practical experience Reflexive thinking. Therefore, this part of the content is also a key framework for the media 
to convince the public. In the layout of the magazine content and the deconstruction of the visual meaning of the topic, the text plays a profound interpretive role. The pictures appear as auxiliary elements. On the one hand, it attracts readers' attention in the layout design and on the other hand due to the particularity of the topic or the angle of interpretation. Give the image space to express, the image appears as " visual evidence " to help the public interpret the textual information and meaning. This framework focuses on introducing to the public what " plastics " is, the process of development and evolution from ancient times to the present, and how it constitutes a risk threat to the environment, society, and human development. The intention is to use a descriptive framework to deconstruct complex, uncertain, hidden and diverse environmental risk issues in the form of storytelling into common-sense knowledge that the general public is willing to know and understand, and to deepen the public's understanding of plastics from the ontological level. Understanding of the formation, development and impact of pollution issues.

Third, at the action level, it emphasizes the construction of " methodology ". Thinking about actions through the " cognition" and " understanding" of risks, but what and how to effectively reduce or block the impact and harm of risk events is an indispensable link for the media to spread risk issues to the public , Only by allowing the public to develop environmentally friendly behaviors can the earth's ecology be truly protected. In the visual layout of this part of the text, the text description and narration play a leading role, and the pictures are only used as supplementary elements to play an active typographic style and relieve the visual fatigue of the public's cognition of complex scientific knowledge. The text description aims to use a " practical framework " to inform the public about the difficulty of plastic pollution control at this stage and the risk response initiative of human beings, and through the description of the national strategy to successfully control plastic pollution risks, so that the society and the public can understand Understand and give a page of content at the end of the special issue to provide a reference for the public to take to alleviate the environmental crisis of plastic pollution. It is intended to persuade the public to realize that the risk of plastic pollution can be mitigated and solved through environmental protection behaviors. This disassembly and reconstruction of risk issues enhances the public's willingness and ability to participate in behavior, and achieves the effect of public dissemination and persuasion of plastic pollution environmental and scientific risk issues.

Finally, the main limitation of this research is that the actual impact of the use of image symbols and visual rhetoric frameworks in the communication of environmental risks on the public's risk perception and behavioral participation has not been empirically studied in this research, because the public's environmentally friendly behavior has The prevention and management of risks are of extremely important significance. It is recommended that in future research, questionnaire surveys, in-depth interviews or experimental methods should be considered to test the public's cognition and behavior to verify the actual effects of image symbols and visual rhetoric frameworks.

\section{REFERENCES}

Adam, B. (1998). Timescapes of modernity: The environment and invisible hazards. London, UK: Psychology press.

Ahern, L., Bortree, D. S., \& Smith, A. N. (2013). Key trends in environmental advertising across 30 years in National Geographic magazine. Public understanding of science, 22(4), 479-494.

Anne DiFrancesco, D., \& Young, N. (2011). Seeing climate change: The visual construction of global warming in Canadian national print media. cultural geographies, 18(4), 517-536.

Antilla, L. (2005). Climate of scepticism: US newspaper coverage of the science of climate change. Global environmental change, 15(4), 338-352.

Baran, S., \& Davis, D. (2011). Mass communication theory: Foundations, ferment, and future. CA: Nelson Education.

Barthes, R. (1977). Rhetoric of the Image. London: Fontana.

Beck, U. (1992). Risk society: Towards a new modernity. London, UK: Sage.

Beck, U. (2002). The terrorist threat: World risk society revisited. Theory, Culture \& Society, 19(4), 39-55.

Berger, A. A. (2017). Media analysis techniques. Los Angeles, CA: Sage Publications.

Carvalho, A., \& Burgess, J. (2005). Cultural circuits of climate change in UK broadsheet newspapers, 1985-2003. Risk Analysis: An International Journal, 25(6), 1457-1469.

Chou, K. (1998). Modernity and risk society. Taiwanese Journal of Sociology, 21, 89-129.

Cottle, S. (2000). TV news, lay voices and the visualisation of environmental risks. Environmental risks and the media, 29-44.

Dias, B., \& Lovejoy, T. E. (2012). Impacts of marine debris on biodiversity: current status and potential solutions. $C B D$ Technical Series, 67, 11-26. 
Dias, B. d. S. (2016). Marine debris: understanding, preventing and mitigating the significant adverse impacts on marine and coastal biodiversity. CBD Technical Series, 83, 17-19.

Doyle, J. (2007). Picturing the clima (c) tic: Greenpeace and the representational politics of climate change communication. Science as culture, 16(2), 129-150.

Dunlap, R. E., Bechtel, R., \& Churchman, A. (2002). Environmental sociology. Handbook of environmental psychology, 2, 160-171.

Elliot, N. L. (2013). Mediating nature. London: Routledge.

Gamson, W. A., \& Modigliani, A. (1989). Media discourse and public opinion on nuclear power: A constructionist approach. American journal of sociology, 95(1), 1-37.

Hansen, A., \& Machin, D. (2013). Researching visual environmental communication. Environmental Communication: A Journal of Nature and Culture, 7(2), 151-168.

Hill, C. A., \& Helmers, M. (2012). Defining visual rhetorics. NY: Routledge.

Howenstine, E. (2005). Environmental reporting: Shift from 1970 to 1982. Journalism Quarterly, 64, 842-846.

Iyer, A., \& Oldmeadow, J. (2006). Picture this: Emotional and political responses to photographs of the Kenneth Bigley kidnapping. European Journal of Social Psychology, 36(5), 635-647.

Joffe, H. (2008). The power of visual material: Persuasion, emotion and identification. Diogenes, 55(1), 84-93.

Johnson, B. B., \& Covello, V. T. (2012). The social and cultural construction of risk: Essays on risk selection and perception (Vol. 3): Springer Science \& Business Media.

Kershaw, P. J. (2016). Marine plastic debris and microplastics-Global lessons and research to inspire action and guide policy change. Retrieved 2021.01.10 http://ec.europa.eu/environment/marine/good-environmental-status/descriptor10/pdf/Marine_plastic_debris_and_microplastic_technical_report_advance_copy.pdf

Kinsella, W. J. (2002). Problematizing the distinction between expert and lay knowledge. Atlantic Journal of Communication, 10(2), 191-207.

Li, Y., \& Wang, S. (2015). A niche analysis on the competition among print magazine, online magazine and mobile magazine. The Journal of Information Society, 28, 35-65.

Liu, T. (2014). Symbol Reconstruction and Rhetoric Practice of Environmental Public Events -- Semiotics analysis of Lanzhou tap water pollution incident. Journalism Bimonthly, 6, 24-31.

Liu, T. (2016). China in Western Data Journalism: A Visual Rhetoric Analytical Framework. Journalism and Communication Studies, 2(7), 5-28.

Liu, T. (2017). Media, Space, and Event: The "grammar" of seeing and visual rhetoric methodology. Nanjing Journal of Social Sciences, 9, 100-109.

Liu, T. (2018). Function of visual rhetoric: Three rhetoric view of the visual studies. Journal of China University of Geosciences ( Social Sciences Edition ), 18(2), 155-165.

Macnaghten, P., \& Urry, J. (1997). Contested natures. London: Sage.

McQuail, D. (1987). Mass communication theory: An introduction. London: Sage Publications.

Mead, G. H. (1934). Mind, self and society (Vol. 111). Chicago: Chicago University Press.

Meisner, M. S., \& Takahashi, B. (2013). The nature of Time: How the covers of the world's most widely read weekly news magazine visualize environmental affairs. Environmental Communication: A Journal of Nature and Culture, 7(2), 255-276.

Peeples, J. (2011). Toxic sublime: Imaging contaminated landscapes. Environmental Communication: A Journal of Nature and Culture, 5(4), 373-392.

Perlmutter, D. D. (1998). Photojournalism and foreign policy: Icons of outrage in international crises: Praeger Publishers.

Pezzullo, P. C., \& Cox, R. (2017). Environmental communication and the public sphere: Sage Publications.

Remillard, C. (2011). Picturing environmental risk: The Canadian oil sands and the National Geographic. International Communication Gazette, 73(1-2), 127-143.

Rice, J. (2004). A critical review of visual rhetoric in a postmodern age: complementing, extending, and presenting new ideas. Review of Communication, 4(1-2), 63-74. 
Schoenfeld, A. C., Meier, R. F., \& Griffin, R. J. (1979). Constructing a social problem: the press and the environment. Social problems, 27(1), 38-61.

Simonsen, J., Bærenholdt, J. O., Büscher, M., \& Scheuer, J. D. (2010). Design research: Synergies from interdisciplinary perspectives. London: Routledge.

Smith, N. W., \& Joffe, H. (2009). Climate change in the British press: The role of the visual. Journal of Risk Research, 12(5), 647-663.

Soper, K. (1995). What is nature?: Culture, politics, and the non-human. UK: Oxford University Press.

Todd, A. M. (2010). Anthropocentric distance in National Geographic's environmental aesthetic. Environmental Communication, 4(2), 206-224.

Urry, J. (1992). The tourist gaze and theEnvironment'. Theory, Culture \& Society, 9(3), 1-26.

Wahlberg, A. A., \& Sjoberg, L. (2000). Risk perception and the media. Journal of Risk Research, 3(1), 31-50.

Williams, R. (1975). The country and the city (Vol. 423). USA: Oxford University Press.

Yang, Y., \& Hsu, M. (2012). Perception and communication of environmental risk: A case study of situational publics regarding global warming. Chinese Journal of Communication Research, 22, 169-210. 